\title{
Indirect search for dark matter in M 31 with the CELESTE experiment
}

\author{
J. Lavalle ${ }^{1, \star}$, H. Manseri ${ }^{2}$, A. Jacholkowska ${ }^{1}$, E. Brion ${ }^{3}$, R. Britto ${ }^{1}$, P. Bruel ${ }^{2}$, J. Bussons Gordo ${ }^{1}$, D. Dumora ${ }^{3}$,

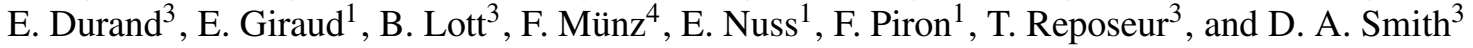 \\ 1 Laboratoire de Physique Théorique et Astroparticules, CNRS-IN2P3/Université de Montpellier II, 34095 Montpellier, France \\ e-mail: lavalle@in2p3.fr \\ 2 Laboratoire Louis Leprince-Ringuet, CNRS-IN2P3/École Polytechnique, 91128 Palaiseau, France \\ 3 Centre d'Etudes Nucléaires de Bordeaux-Gradignan, CNRS-IN2P3, 33175 Bordeaux, France \\ ${ }^{4}$ Laboratoire d'Astroparticule et Cosmologie, CNRS-IN2P3/Collège de France, 75231 Paris, France
}

Received 11 October 2005 / Accepted 5 January 2006

\section{ABSTRACT}

Context. If dark matter is made of neutralinos, annihilation of such Majorana particles should produce high energy cosmic rays, especially in galaxy halo high density regions like galaxy centres.

Aims. M 31 (Andromeda) is our nearest neighbour spiral galaxy, and both its high mass and its low distance make it a source of interest for the indirect search for dark matter through $\gamma$-ray detection.

Methods. The ground based atmospheric Cherenkov telescope CELESTE observed M 31 from 2001 to 2003, in the mostly unexplored energy range $50-500 \mathrm{GeV}$.

Results. These observations provide an upper limit on the flux above $50 \mathrm{GeV}$ around $10^{-10} \mathrm{~cm}^{-2} \mathrm{~s}^{-1}$ in the frame of supersymmetric dark matter, and more generally on any gamma emission from M 31 .

Key words. gamma ray: observations - cosmology: dark matter - galaxies: spiral

\section{Introduction}

The presence of dark matter in the Universe has been known for decades. Since early measurements in galaxy clusters (Zwicky 1937), the mass distribution of the Universe has been studied at different scales with a focus on dynamical effects by means of galaxy star rotation curves, large-scale galaxy cluster dynamics (Ostriker et al. 1974). Recent developments in observational techniques in cosmology have resulted in independent estimates of the matter content of the Universe $\Omega_{\mathrm{m}} h^{2}$, through large-scale structure surveys (Hawkins et al. 2003; Loveday et al. 2002) and measurements of the cosmic microwave background radiation (the most recent being the WMAP mission Spergel et al. 2003). Given standard cosmology, all suggest that most of the matter in the Universe is dark, cold and nonbaryonic. Such hypotheses have led to the construction of the Cold Dark Matter (CDM) paradigm (Blumenthal et al. 1984): dark matter would be made of Weakly Interacting Massive Particles (WIMPs) which are neutral, stable and originating from the Big Bang era (Lee \& Weinberg 1977).

^ Present address: Centre de Physique des Particules de Marseille, CNRS-IN2P3/Université Aix-Marseille II, 13288 France.
Supersymmetric theories (SUSY) (see for instance Nilles 1984) offer an excellent WIMP candidate (Goldberg 1983), the neutralino, which is a mixture of the superpartners of the neutral Higgs bosons and of the electroweak gauge bosons. We will not discuss the case of extra-dimension phenomenological theories, which also provide interesting candidates (Servant \& Tait 2003).

The nature of dark matter is presently probed in both direct searches, by means of underground experiments that could detect elastic interactions of neutralinos with nuclei, and indirect searches using ground based or satellite telescopes to detect cosmic rays (gamma, leptons or hadrons) created by neutralino pair annihilations in galactic or extragalactic media (for review, see Bergström 2000).

These different types of searches, together with collider experiments, are necessary to constrain in a wider view the quantum nature of dark matter, because they allow either additional or complementary surveys of the particle model parameter spaces.

Searching for WIMP annihilation signatures with ground based $\gamma$-ray telescopes leads to the question of the choice of targets. A good candidate will have a large amount of dark matter, and combine as big a density as possible, as small a distance 
from us as possible, and finally, will transit at high elevation in the experimental sky. The Galactic centre is a prime candidate except for being too close to the horizon for CELESTE, which is in the northern hemisphere. Instead, we have chosen M 31 and the Draco dwarf galaxy for our searches. M31, which is the nearest giant spiral galaxy, is very massive $\left(\sim 10^{12} M_{\odot}\right)$, and its star rotation curve indicates a large amount of dark matter. Draco, a neighbour dwarf spheroidal galaxy dominated by a dark component (Kleyna et al. 2001), is also a very good candidate but our attempts to study it were foiled by bad weather.

In this paper, we present the result of searches for $\gamma$-ray emission from M31 with the CELESTE telescope. In Sect. 2, we review the predictions made for the observations (more details in Falvard 2004, hereafter F04), for which we considered CDM in the frame of minimal supergravity (mSUGRA) phenomenology. The impact of the astrophysical modelling is briefly revisited, as well as possible consequences of nonstandard cosmologies. Focusing on the experimental techniques, we present in Sect. 3 the method we use to search for a $\gamma$-ray signal, with explicit comparisons to Crab data. In the absence of a detection, $2 \sigma$ confidence level limits are computed for all studied SUSY models.

\section{Gamma-ray flux predictions for supersymmetric annihilating dark matter in M 31}

Under the assumption that neutralinos have an isotropic and homogeneous velocity distribution, which is likely to be the case in halo centres where the WIMP velocity is expected to be low, the averaged $\gamma$-ray flux due to their annihilation, integrated above an energy threshold $E_{\text {th }}$ and within the solid angle $\Delta \Omega$ can be written as:

$$
\begin{aligned}
\Phi\left(E_{\mathrm{th}}\right) & =\frac{1}{4 \pi} \frac{N_{\gamma}\left(E_{\mathrm{th}}\right)\langle\sigma v\rangle}{2 m_{\chi_{0}}^{2}} \int_{\Delta \Omega(\theta)} \int_{\text {l.o.s. }} \rho^{2}(s) \mathrm{d} s \mathrm{~d} \Omega \\
& \equiv \frac{1}{4 \pi} \frac{N_{\gamma}\left(E_{\mathrm{th}}\right)\langle\sigma v\rangle}{2 m_{\chi_{0}}^{2}} \Sigma(\theta) .
\end{aligned}
$$

We thus decouple astrophysics modelling from SUSY contributions. The first part of the right hand term is related to particle physics, via the thermally averaged product of the cross section $\sigma$ with the velocity $v$ producing $N_{\gamma}\left(E_{\mathrm{th}}\right)$ photons of energy $E>E_{\text {th }}$, and the neutralino mass $m_{\chi_{0}}$. The second term refers to the (squared) halo density profile $\rho$ integrated within an experimental field of view of angular radius $\theta$ along the line of sight $\mathrm{d} s$. At the same time, we define $\Sigma(\theta)$ as the astrophysical factor of the flux.

As $\gamma$-rays result mainly from hadronization of annihilation final states (mainly quarks and gauge bosons), their spectral shape mainly takes its origin in the decay of $\pi^{0}$. It has been shown by several authors (see for instance Bergström et al. 1998, or Tasitsiomi et al. 2002) that such a spectrum can be fitted or modelled with respect to the neutralino mass. Therefore, the differential spectrum above a threshold energy $E_{\text {th }}$ can be written as follows:

$$
\frac{\mathrm{d} \Phi}{\mathrm{d} E}\left(E>E_{\mathrm{th}}\right) \equiv \Phi\left(E_{\mathrm{th}}\right) \times f\left(E, m_{\chi_{0}}\right)
$$

where $f\left(E, m_{\chi_{0}}\right)$ is the spectral shape derived from the SUSY model and normalized such that $\int_{E_{\mathrm{th}}}^{\infty} f\left(E, m_{\chi_{0}}\right) \mathrm{d} E=1$, so that $\Phi\left(E_{\mathrm{th}}\right)$ is the integrated spectrum above an energy threshold $E_{\text {th }}$. This expression will be useful when we interpret the M31 data collected by CELESTE.

\subsection{Halo modelling}

M31 is a late-type Sb spiral galaxy, which lies at a distance of about $675 \mathrm{kpc}$, and is observable from the Northern hemisphere $\left(\mathrm{RA}=10.68^{\circ}, \mathrm{DEC}=41.27^{\circ}\right)$. A study by Braun (1991), based upon the analysis of HI data and a modelindependent reconstruction of the velocity field, showed that the star rotation curve arises naturally by considering two optically traced mass components: a bulge, with a total mass of $(7.8 \pm 0.5) \times 10^{10} M_{\odot}$, and a disk of $(1.22 \pm 0.05) \times 10^{11} M_{\odot}$ within $28 \mathrm{kpc}$. Nevertheless, it seems that the star mass-to-light ratios used in this paper, $\Upsilon_{\text {bulge }}=6.5$ and $\Upsilon_{\text {disk }}=6.4$ (solar units in blue band), are over-estimated (cf. F04).

By lowering the bulge and disk contributions, that is $\Upsilon_{\text {bulge }}=3.7$ and $\Upsilon_{\text {disk }}=2.5$ as indicated by F04, we assumed that a dark halo significantly accounts for the gravitational potential. Let us consider the following CDM density profile:

$\rho_{\mathrm{CDM}}(r)=\rho_{0}\left(\frac{r_{0}}{r}\right)^{\gamma}\left(\frac{r_{0}^{\alpha}+a^{\alpha}}{r^{\alpha}+a^{\alpha}}\right)^{\epsilon}$,

where $r_{0}$ usually stands for a core radius and $a$ a scale length. An Navarro-Frenk-White profile (Navarro et al. 1996), i.e. with $\gamma=1, \alpha=1$ and $\epsilon=2$, fits the rotation curve, and has its parameters entirely determined by the previous mass-to-light ratios. The fitted values are $\rho_{0} \sim 0.03 \mathrm{GeV} \mathrm{cm}^{-3}, r_{0} \sim 20 \mathrm{kpc}$ and $a \sim 5 \mathrm{kpc}$. The resulting contribution to the $\gamma$-flux within CELESTE's field of view $\left(\theta=5 \mathrm{mrad} \sim 0.3^{\circ}\right.$, corresponding to a $3.5 \mathrm{kpc}$ radius) has been calculated:

$\Sigma(\theta=5 \mathrm{mrad})=3 \times 10^{19} \mathrm{GeV}^{2} \mathrm{~cm}^{-5}$.

This result depends strongly on the central tail of the dark halo, but is rather conservative since calculated with a $r^{-1}$ profile.

\subsection{Probing the SUSY parameter space}

We choose the minimal supergravity framework (mSUGRA) to scan over the SUSY parameter space. In this frame, a SUSY model can be defined at the unification scale with 5 parameters: the unified scalar mass $m_{0}$, the unified gaugino mass $m_{1 / 2}$, the Higgs vacuum expected value ratio $\tan (\beta)$, the unified trilinear coupling $A_{0}$ and the sign of the mixing parameter of the Higgs superfields $\mu$. We use an interface between the public codes Suspect (Djouadi et al. 2002) and DarkSusy (Gondolo et al. 2004) to compute SUSY masses, annihilation rates and relic densities for various random models. The constraints on these models come from standard accelerator limits, and we select a rather large range for the relic density $\left(\Omega_{\chi_{0}} h^{2} \in[0.05,0.14]\right)^{1}$,

\footnotetext{
${ }^{1}$ The upper limit is given by the WMAP result plus three sigma. Higher values for the relic density are not that interesting because they correspond to lower values of the annihilation cross-section.
} 


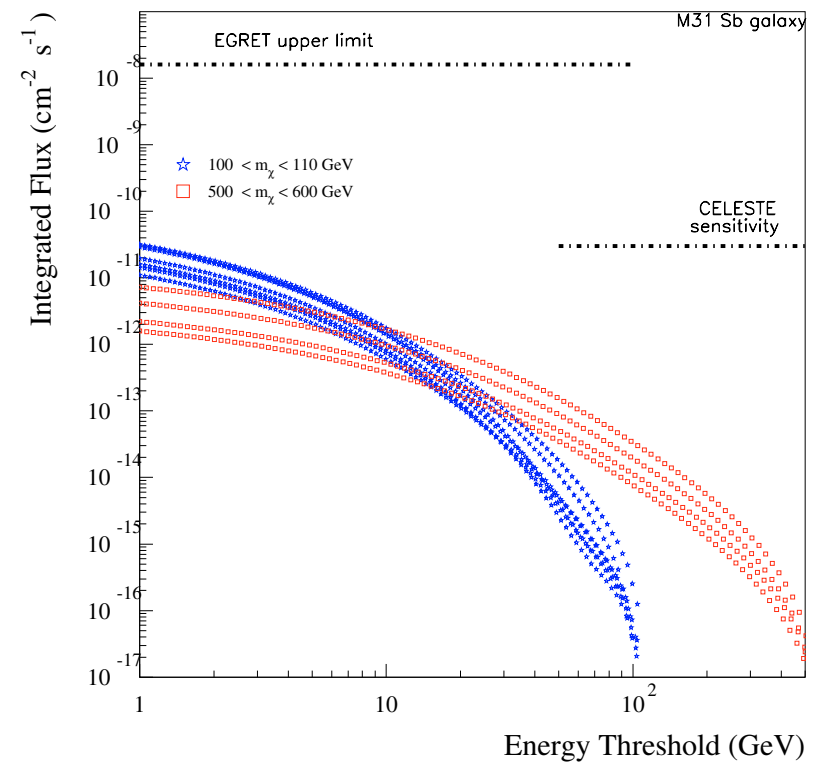

Fig. 1. Integrated expected $\gamma$-flux from M31 as a function of the energy threshold, for two selections of low (100-110 GeV) and high (500-600 GeV) neutralino masses $\left(\Omega_{\chi_{0}} h^{2} \in[0.086,0.14]\right)$, showing the mass-dependence of the flux, and the complementarity between EGRET and CELESTE.

according to the WMAP measurement $\Omega_{\chi_{0}} h^{2}=0.113 \pm 0.009$ (Bennett et al. 2003).

Combining the resulting $\gamma$-spectrum with the astrophysical factor $\Sigma$, we calculate the integrated flux as a function of the threshold energy, as shown in Fig. 1, in which we have selected only two groups of WMAP compatible neutralinos (respectively small and high masses) in order to exhibit the massdependence of the expected flux. The SUSY models plotted there are characterized by a high value of $\tan \beta$ (typically $>30$ ), for which the production of $\gamma$-rays is more efficient (due to high branching ratio in b quarks). On the same plot, we represent the upper limit provided by the EGRET collaboration (Blom et al. 1999) and CELESTE's sensitivity, $\sim 6 \times 10^{-11} \mathrm{ph} \mathrm{cm}^{-2} \mathrm{~s}^{-1}$, estimated for $50 \mathrm{~h}$ of observation. This figure illustrates the massand/or-energy experimental complementarities.

\subsection{Other contributions}

Although the predicted fluxes are low $\left(\sim 10^{-13} \mathrm{~cm}^{-2} \mathrm{~s}^{-1}\right.$ at a threshold of $\sim 50 \mathrm{GeV}), \sim 3$ orders of magnitude smaller than for the Crab nebula (therefore far from CELESTE's sensitivity), several effects could enhance them.

First of all, dark matter substructures, the so-called clumps, arise naturally in the hierarchical scheme of galaxy formation, and simulations of the non-linear regime of collapse allow a semi-theoretical study of their statistics and structure (Moore et al. 1999). Such local overdensities should induce extra neutralino annihilations, and translate to an additional factor to the flux. Although it is rather difficult to estimate how clumpy the galaxies remain today, this enhancement factor was until recently supposed to be smaller than $\sim 10$ (Stoehr et al. 2003). Nevertheless, a recent study by Diemand et al. (2005) suggests that about $50 \%$ of a Milky-Way-like galaxy mass is possibly bound to dark matter substructures, whose mass range spreads from $10^{-6}$ up to $10^{7} M_{\odot}$. The authors claim that about $\sim 10^{15}$ of such substructures may have survived against gravitational disruption, leading to a boost factor of over two orders of magnitude compared to the smooth contribution. M31 being very similar to our galaxy, this statement should also stand for that source.

As another possible astrophysical effect, the supermassive black hole at the centre of M 31 could raise the central halo profile up due to adiabatic accretion (Gondolo \& Silk 1999). There are other interesting mechanisms involving baryons to enhance the dark matter density. Gnedin et al. (2004) sketch such responses of halos to condensation of baryons, while Bertone et al. (2005) propose a possible enhancement of the WIMP annihilation rate due to the presence of intermediate-mass black holes.

Beside those astrophysical effects, some recent developments in the frame of theoretical cosmology have focused on the quintessence scheme (Caldwell et al. 1998) to solve the socalled coincidence problem (the fact that $\Omega_{\Lambda} \sim \Omega_{\text {matter }}$ today). Such a quintessential field could undergo a kination regime in the early universe (Salati 2003), so that its kinetic energy dominates over its potential. In this regime, the expansion rate of the universe is enhanced and the thermal history of neutralinos is consequently modified: the decoupling of neutralinos can take place more rapidly at earlier times. Therefore, the WMAP constraint leads to a higher neutralino annihilation cross-section. This means that this phenomenon rehabilitates SUSY models for which relic densities are too low, when calculated in standard cosmology. Salati (2003) shows that the relic density enhancement can be parameterized by:

$\Omega_{\chi_{0}} \rightarrow \tilde{\Omega}_{\chi_{0}} \simeq 1000\left(\frac{m_{\chi_{0}}}{100 \mathrm{GeV}}\right) \sqrt{\eta_{0}} \Omega_{\chi_{0}}$ with $\eta_{0} \leq 0.3$

$\eta_{0} \equiv \rho_{\Phi, 0} / \rho_{\gamma, 0}$, where the 0 -index refers to a temperature of $1 \mathrm{MeV}$, and $\rho_{\Phi}$ (respectively $\rho_{\gamma}$ ) is the quintessence (photon) energy density. The upper limit on $\eta_{0}$ comes from Big Bang Nucleosynthesis stages that should not be perturbed by the kination regime (Yahiro et al. 2002).

According to this cosmology, higher annihilation rate models are required, which therefore means that the $\gamma$-ray production is enhanced. This effect appears in the final results showed in Fig. 7, for a small sample of selected SUSY models.

Finally, we emphasize another interesting effect coming from Affleck-Dine baryogenesis in SUSY (Fujii et al. 2004), which yields natural matter-antimatter asymmetry in the early universe. In such a scenario, meta-stable particles result from oscillations in flat directions of the scalar potential, carrying baryon and/or lepton number, namely Q-balls. These Q-balls can have a lifetime long enough to decay after the freeze-out of neutralinos. This induces a non-thermal production of neutralinos, and thus enhances their relic density. This also requires, as previously, higher neutralino annihilation rates to not overclose the universe.

Therefore, although standard conservative predictions are not that optimistic, all these putative contributions may increase the $\gamma$-flux from M31 significantly. This further 
motivates observations of such a source with CELESTE, keeping in mind that CDM could be something besides SUSY.

\section{Observations of M 31 with CELESTE}

\subsection{The CELESTE experiment}

CELESTE (Paré et al. 2002; de Naurois et al. 2002) is an atmospheric Cherenkov telescope detecting $\gamma$-rays above $\sim 50 \mathrm{GeV}$ (the experiment shut down in June 2004). Reaching such a low energy threshold required a large light collection area, achieved by exploiting the mirrors of a solar plant. These mirrors are used to sample the arrival time and photon flux of the Cherenkov wavefront generated by atmospheric showers initiated by cosmic rays at many points in the light pool. In contrast to the imaging technique (Weekes 1988) the sampling technique uses information on the shape of the wavefront for hadron rejection, as described below.

The CELESTE experiment uses 53 heliostats (40 until 2001) of the Thémis former solar plant (French Pyrénées, $42.50^{\circ} \mathrm{N}, 1.97^{\circ} \mathrm{E}$, altitude $\left.1650 \mathrm{~m}\right)$. Each heliostat $\left(54 \mathrm{~m}^{2}\right)$ reflects the light onto the secondary optics, located at the top of a $100 \mathrm{~m}$ tower, focussing the light onto a single photomultiplier (PMT) for each heliostat. The PMT signals are sent to the trigger electronics and to the data acquisition system where they are digitized by $\sim 1 \mathrm{GHz}$ flash analog-to-digital converters (FADCs).

The mean altitude of the maximum Cherenkov emission for $\gamma$-ray induced showers is around $11 \mathrm{~km}$ above the site. The heliostats are aimed at this altitude in the direction of the source under study to enhance light collection. The observations are made in the On-Off tracking mode: the observation of the source (On) is followed or preceded by an observation at the same declination offset in right ascension by $20 \mathrm{~min}$. The latter is used as a reference for the cosmic-ray background and the signal is given by the difference between On and Off, after analysis cuts.

M 31 is a special source for CELESTE in that its blue magnitude is about 4.3 , and $\sim 5.4$ if integrated in the \pm 5 mrad field of view of CELESTE (de Vaucouleurs 1958). Hence, pointing On-source increases the PMT illumination compared to the Off-source data. The same problem arises to a lesser degree for the study of the blazar Mrk421, due to the presence of the $m_{B}=6.16$ star $51 \mathrm{U}$ Ma in the same field-of-view. On-off illumination differences introduce biases at the trigger level and in the recorded data which fake a signal if not handled properly. We remove these biases by "padding" the Off-source data with extra background light as part of the data analysis, and by making a pulseheight cut $10 \%$ above the hardware trigger threshold, as described in (de Naurois et al. 2002) and updated in (Manseri 2004).

In the following, all Monte Carlo simulations are performed at the transit position of sources in the Thémis sky, unless specified. We have used the atmospheric shower simulator Corsika (Heck et al. 1998) for our Monte Carlo studies. Moreover, stellar photometry studies using the PMT anode currents provided an improved description of our optics in the detector simulation (Smith \& Brion 2004).

\subsection{Hadron rejection}

In our energy range, the Cherenkov wavefront from $\gamma$-ray induced showers is, on average, more spherical than for showers initiated by hadrons (that is, by charged cosmic rays, mainly protons). The CELESTE field of view is small compared to the angular extent of the showers, which lessens the difference, but efficient hadron rejection is still possible.

Just above the trigger threshold, the Cherenkov signal for many heliostats is comparable to the fluctuations of the night sky background light, so we sum all the signals instead of using each individually. Summing the signals implies compensating for the propagation delays, which requires knowledge of the shower core position when assuming a spherical Cherenkov wavefront. As described in Manseri (2004), this is done by scanning the plane at $11 \mathrm{~km}$ and evaluating the ratio $H / W$ for each position of the scan, where $H$ and $W$ are the height and the width of the summed signal. The largest value of the ratio $H / W$ yields our measure of the shower core position. The sphericity of the wavefront is estimated by how much the ratio $H / W$ decreases when estimated $200 \mathrm{~m}$ away from the shower core position. This relative decrease, called $\xi$, is shown in Fig. 2-top for an Off observation and for a simulation of a $\gamma$-ray spectrum. As expected, because of their Cherenkov wavefront sphericity, $\gamma$-ray showers have lower $\xi$ values than hadronic showers.

The On-Off difference of this relative decrease is shown in Fig. 2-bottom for a sample of Crab nebula data, which was taken between 2002 and 2004 with the same experimental setup as for M 31. It exhibits a clear excess due to the $\gamma$-ray emission from the Crab nebula. Requiring $\xi<0.35$ leads to a $13.5 \sigma$ excess and a sensitivity of $6.5 \sigma / \sqrt{h}$.

\subsection{Observations, data selection and signal searches}

M31 was observed with CELESTE from 2001 to 2003, and 68 On-Off pairs were collected ( $22 \mathrm{~h}$ of On-source data). Nevertheless, variations in atmospheric conditions are known to cause systematic shifts in the On-Off difference, so we applied a selection based on criteria requiring stable detector operation (characterized by PMT anodic current and trigger rate stability). This selection reduced the data set to $6.5 \mathrm{~h}$ because of bad weather conditions at Thémis since 2001.

The On-Off difference of $\xi$ for the M31 data is shown in Fig. 3-top. No evidence of an excess can be found, and the OnOff difference is $-0.75 \sigma$ when requiring $\xi<0.35$.

SUSY annihilating dark matter could produce soft spectra or peaked signals, so we search for a signal in various energy bands. Our energy reconstruction uses $\gamma$-ray simulations with fixed energies, and is based on the correlation between the total charge recorded with the FADCs and the true (i.e. Monte Carlo) energy, for a given impact parameter. (The shower core position at $11 \mathrm{~km}$, obtained by maximizing $H / W$ as described above, gives the impact parameter on the ground assuming that the gamma ray comes from the source under study.) Figure 4 (top) shows the mean charge per heliostat versus the reconstructed impact parameter, for different $\gamma$-ray energies. We use the charge distributions at each energy and impact 

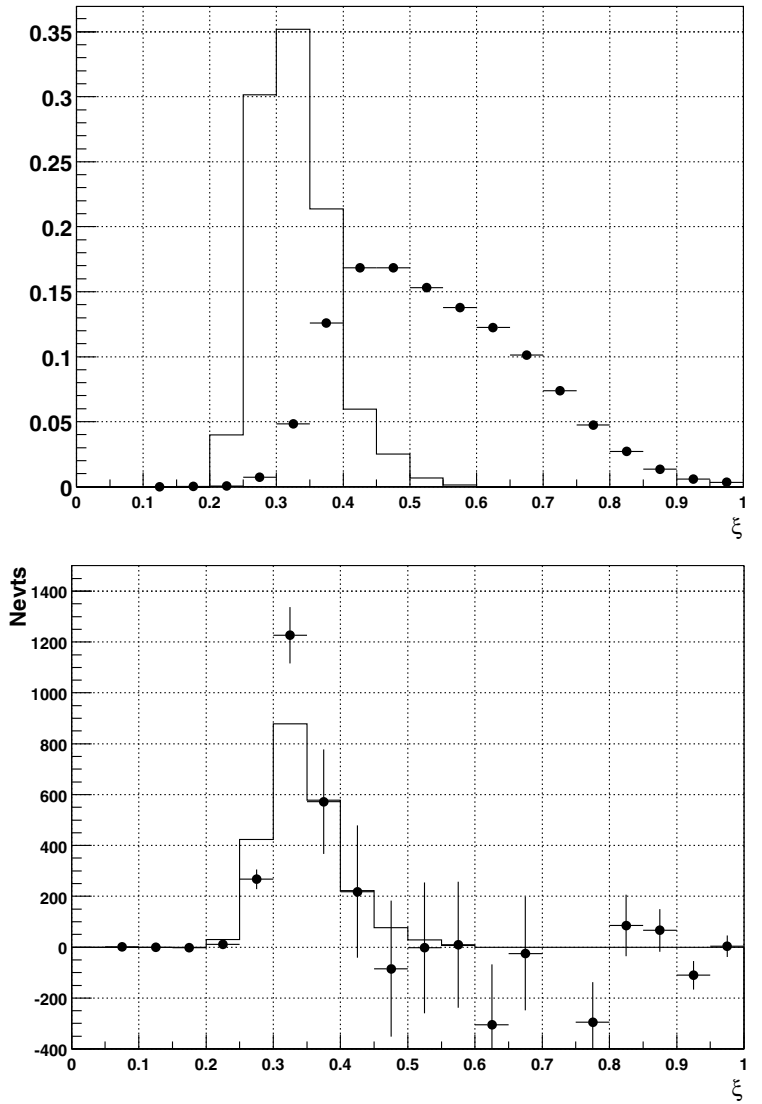

Fig. 2. Top: normalized distributions of the discriminating analysis variable $\xi$ for a simulation of $\gamma$-rays (solid line) from M 31 following a CDM-like spectrum $\left(m_{\chi_{0}}=500 \mathrm{GeV}\right)$, with respect to M31 Off-data (2001-03, black markers). Bottom: number distribution of $\xi$ for a $E^{-2}$-spectrum simulated at the Crab transit (solid line), with respect to the On-Off difference for a data sample of the Crab nebula (taken between 2002 and 2004, black markers with error bars).

parameter to build the inverse function, that is, the function predicting the energy from the observed charge and the reconstructed impact parameter in the range of [0-120] $\mathrm{m}$. To check the function, we inverse it again and compare it to the original points, also shown in Fig. 4 (top). The lower panel of Fig. 4 shows the energy bias and resolution curves obtained from this estimation method. The energy resolution is below $30 \%$ with a small bias of $\sim 5 \%$, adequate to search for an excess in the data.

The On-Off difference of the measured energy distribution, after analysis cuts, is shown in Fig. 3-bottom, in which the $50 \mathrm{GeV}$ binning covers at least one standard deviation of the energy resolution function. No excess has been found. All results are summarized in Table 1, in which a search for an excess within bins of $100 \mathrm{GeV}$, much larger at low energy than the resolution, is presented.

\subsection{Stability and upper limit}

We have also studied the stability of this result, as a check of possible systematic effects. We show in Fig. 5 how the significance remains stable when varying the cut value on the main discriminating variable $\xi$. As a comparison, the same exercise has been done for a Crab dataset (same figure), and the $\gamma$-ray
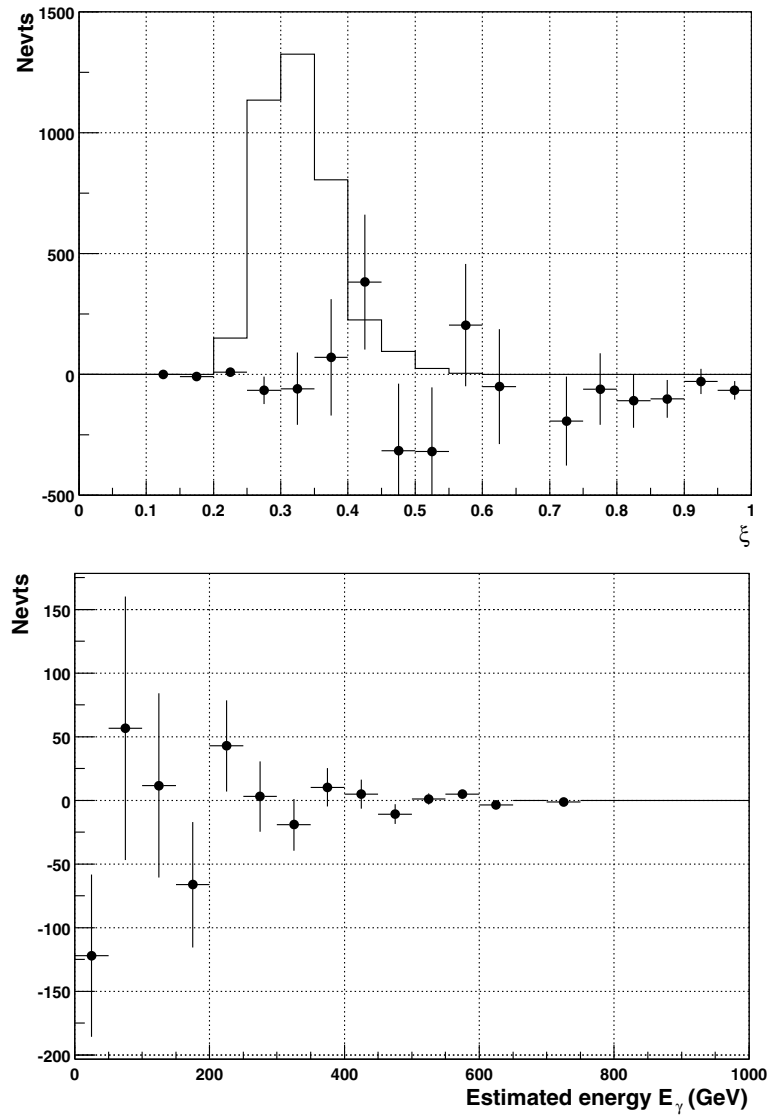

Fig. 3. Top: on-off distribution of $\xi$ for M 31 data (2001-03, black markers with error bars), with respect to the same simulated spectrum as Fig. 2-top (solid line). Bottom: on-off distribution of the estimated energy for all events within analysis cuts.

signal clearly exhibits a bump in significance up to $N_{\sigma} \sim 13.5$ around $\xi<0.35$.

On-Off difference measures the integral over energy of the theoretical spectrum of Eq. (2) convoluted with the effective area, times the observation time. As the statistics collected is compatible with the absence of a signal, we have only measured the background and its fluctuations in direction of M31. This can be translated to an upper limit on a flux coming from that source, given a normalized theoretical spectral shape $f(E)$. For a $N_{\sigma}$ upper limit, and given an experimental energy threshold $E_{\text {th }}$, any integrated flux above this energy should be bounded like:

$\Phi\left(E>E_{\mathrm{th}}\right) \leq N_{\sigma} \frac{\delta N_{\mathrm{bkgd}}}{T_{\mathrm{obs}} \int_{E_{\mathrm{th}}}^{\infty} \mathcal{A}(E) f(E) \mathrm{d} E}\left(\right.$ in $\left.\mathrm{cm}^{-2} \mathrm{~s}^{-1}\right)$,

where $\delta N_{\text {bkgd }} \simeq \sqrt{2 \times N_{\mathrm{Off}}}$ is the measured background rms, $T_{\text {obs }}$ is the total exposure time and $\mathcal{A}(E)$ stands for the energydependent effective detection area. The latter is determined by means of simulations, and is plotted in Fig. 6-top. According to Eq. (2), note that the spectrum $f(E)$ is mass-dependent in case of neutralino annihilation, so that the limit should depend on the mass. For the sake of simplicity, we will use the 
Table 1. Final statistics resulting from M 31 data analysis. The analysis cuts are the following: we impose a software trigger $10 \%$ higher than the hardware one, and we apply a cut $\xi<0.35$ (see text for details); for signal searches in energy bins, we add a selection on the reconstructed impact parameter $-<120 \mathrm{~m}-$ according to the validity range of our energy reconstruction method. No significant excess appears over the whole sample, nor within different ranges of energy.

\begin{tabular}{ccccc}
\hline \hline Analysis level & On-source evts & Off-source evts & On-Off & Significance $\left(N_{\sigma}\right)$ \\
\hline Raw data & 463520 & 462327 & 1193 & -0.59 \\
\hline Analysis cuts & 10615 & 10740 & -125 & -0.75 \\
$E^{\text {meas }}<100 \mathrm{GeV}$ & 6101 & 6167 & -66 & -0.53 \\
$100 \leq E^{\text {meas }}<200 \mathrm{GeV}$ & 3143 & 3197 & -54 & -0.61 \\
$200 \leq E^{\text {meas }}<300 \mathrm{GeV}$ & 870 & 824 & 46 & 1.02 \\
$300 \leq E^{\text {meas }}<400 \mathrm{GeV}$ & 260 & 269 & -9 & -0.35 \\
\hline
\end{tabular}
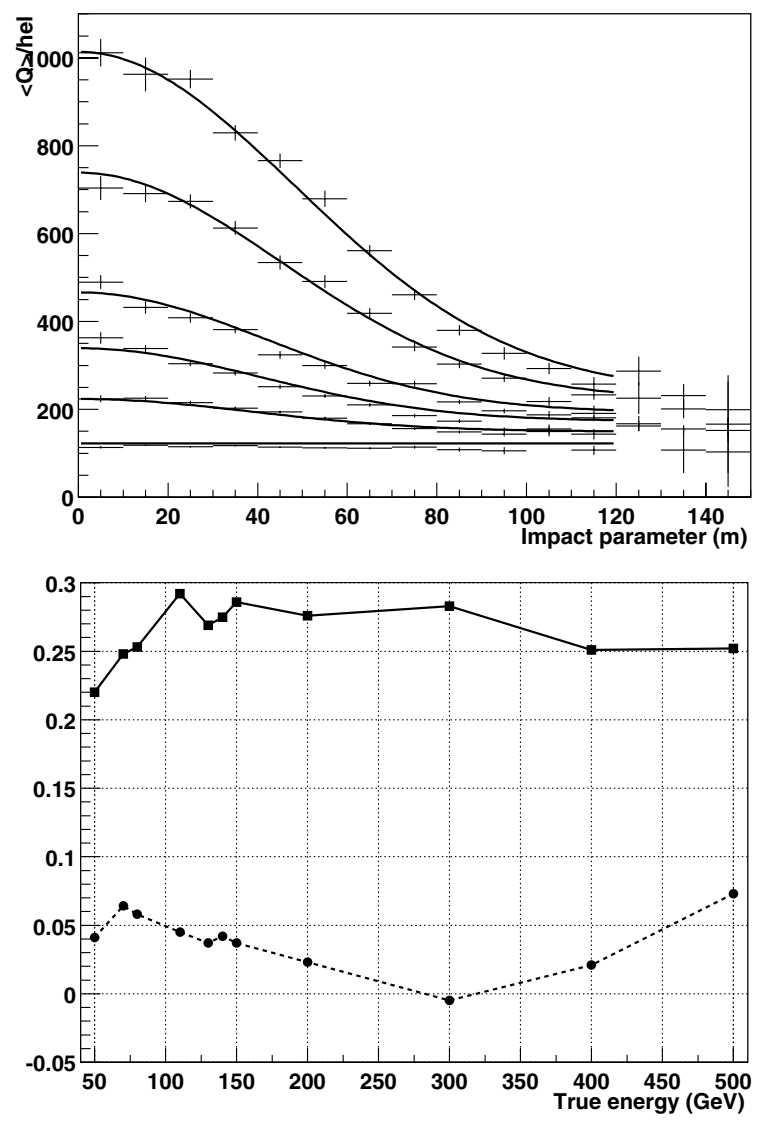

Fig. 4. Top: the crosses are the mean charge per heliostat (arbitrary units) for $\gamma$-rays simulated with energies of 50,100,150, 200, 300 and $400 \mathrm{GeV}$ (bottom-top), versus the reconstructed core position (i.e. the impact parameter). The solid curves show the charge obtained from the functions used to predict the energy. Bottom: resolution (solid upper curve) and bias (lower dashed curve) for the energy reconstruction method with respect to the true energy (same Monte-Carlo set-up as in plot on top).

parametrization given in Tasitsiomi et al. (2002) for the spectral shape, that is:

$$
f(E)=\frac{6 \sqrt{x_{\mathrm{th}}}}{5 m_{\chi_{0}}\left(\sqrt{x_{\mathrm{th}}}-1\right)^{4}}\left(\frac{10}{3}-\frac{5}{12} \frac{3 x^{2}+6 x-1}{x^{3 / 2}}\right)
$$

where $x \equiv E / m_{\chi_{0}}$ and $x_{\mathrm{th}} \equiv E_{\mathrm{th}} / m_{\chi_{0}}$. This spectrum depends on the mass, but assumes that $\gamma$-rays come only from

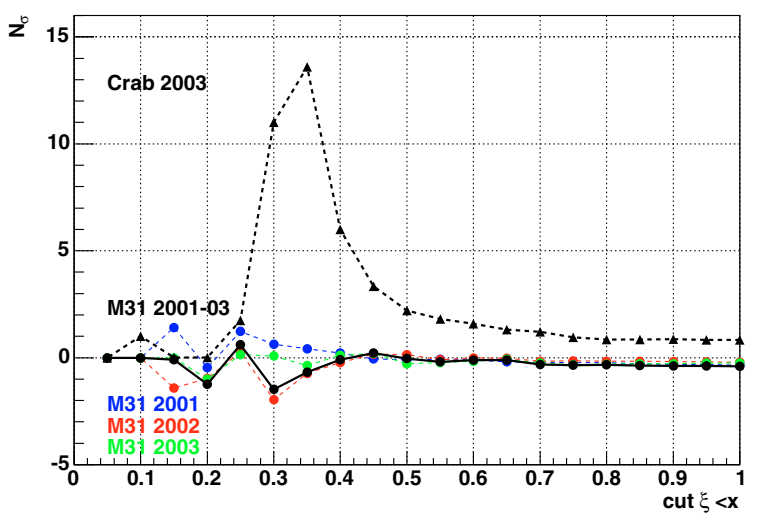

Fig. 5. Significance level according to varying the cut on $\xi$ (after raising the threshold by $10 \%$ ). This figure shows the study of stability of the analysis as well for Crab as for M31 data. A signal evidence is clearly seen for the Crab, whereas the significance level remains flat for M31.

$\pi^{0}$ decay. Therefore, it does not take into account all specificities of SUSY models. Nevertheless, it is sufficient for our purpose. This parametrization is illustrated in Fig. 6-bottom for two masses, 250 and $500 \mathrm{GeV}$, and compared with a $1 / E^{2}$ power law spectrum.

The threshold is set to $50 \mathrm{GeV}$, taken from the effective area shown in Fig. 6 (top). Given this threshold, a flux limit can be computed for each neutralino mass, using Eq. (6). The result is presented in Fig. 7, where the predicted integrated fluxes of $\gamma$-rays above $50 \mathrm{GeV}$ are plotted with respect to neutralino masses. The averaged limit in a mass range of [50-700] GeV lies around $10^{-10} \mathrm{ph} \mathrm{cm}^{-2} \mathrm{~s}^{-1}$. We emphasize that this is the first experimental result in the energy range $50-500 \mathrm{GeV}$, and is complementary to those provided by EGRET (Blom et al. 1999) and HEGRA (Aharonian et al. 2003) observations of M 31.

\section{Discussion and conclusion}

Observations of M 31 with CELESTE provide a $2 \sigma$ upper limit on the $\gamma$-flux above $50 \mathrm{GeV}$, depending on the expected spectrum. This limit, around $10^{-10} \mathrm{ph} \mathrm{cm}^{-2} \mathrm{~s}^{-1}$, is quite far from the SUSY parameter space, but significantly constrains combinations of different enhancement factors discussed in Sect. 2.3 (which are also likely to be excluded by EGRET limits, 

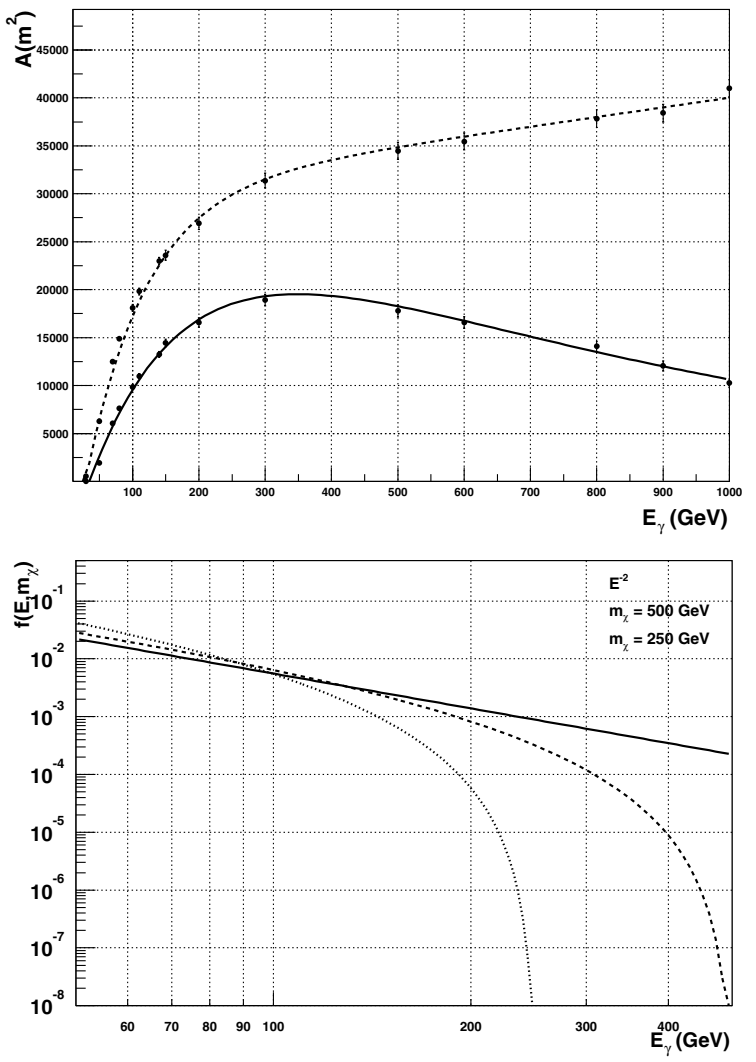

Fig. 6. Top: effective detection area - dashed line for trigger, full after event selection - as a function of the true energy, based on simulations of $\gamma$-rays coming from the transit position of M31. Bottom: normalized spectra between 50 and $500 \mathrm{GeV}$ for a power law of index -2 , and for formula of Eq. (7) with $m_{\chi^{0}}=500$ (dashed curve) and $250 \mathrm{GeV}$ (dotted curve).

depending on the neutralino mass), and also any other model of annihilating dark matter besides SUSY. Whereas these observations have been motivated by indirect searches for SUSY CDM, this result yields a general astrophysical result: the first observation of a spiral galaxy in this energy range, somehow constraining $\gamma$-ray emission from this class of objects.

Any gamma ray detection from a galaxy like M 31 would be difficult to interpret in terms of dark matter annihilation. Spiral galaxies are known sites of non-thermal processes and cosmic ray acceleration, and the relevant physical mechanisms are not yet well understood. In this sense, a Dwarf Spheroidal galaxy like Draco is a very promising source for indirect detection, given it is clearly dominated by the dark matter component. Unfortunately, we have too few data on Draco to perform a relevant analysis.

However, the upcoming generation of $\gamma$-ray instruments will undoubtedly further constrain dark matter models and halo models for various astrophysical sources. These searches are not only complementary to future particle physics experiments, but also very important to understand how the question of dark matter is connected to the particle content of the Universe.

Acknowledgements. Funding was provided by the IN2P3 of the French CNRS and by the Grant Agency of the Czech Republic. We gratefully acknowledge the support of the Regional Council of Languedoc-Roussillon, and of Électricité de France. The technical

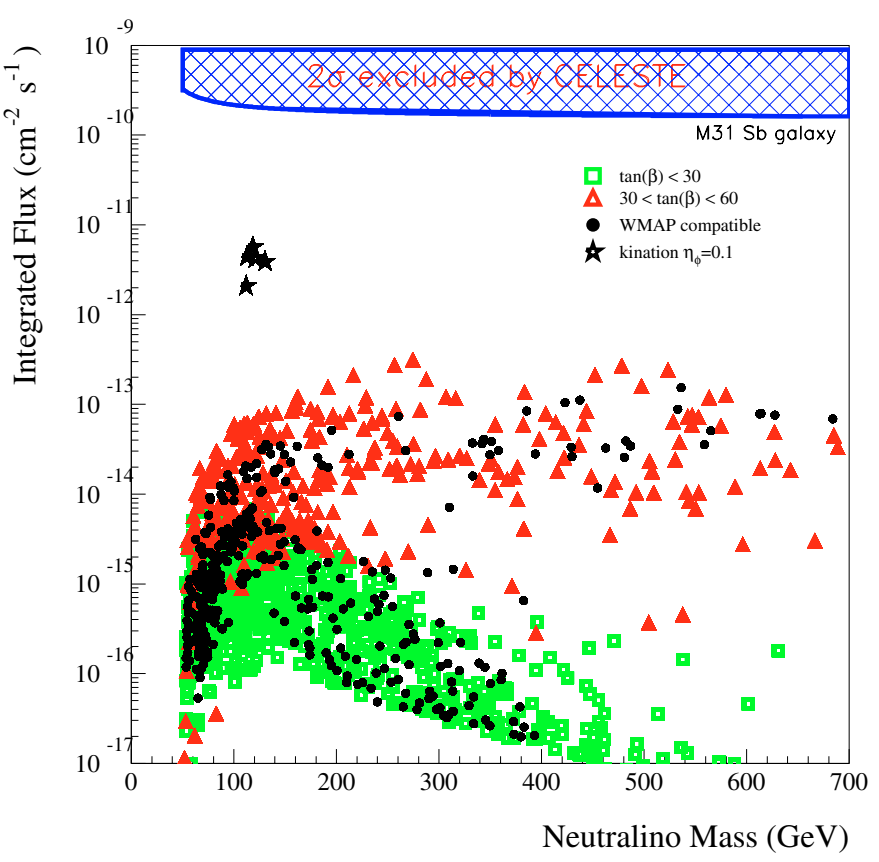

Fig. 7. Integrated flux above $50 \mathrm{GeV}$ as a function of the neutralino mass, for SUSY models with $\Omega h^{2} \in[0.05,0.14]$ (boxes), limited to $[0.086,0.14]$ (full circles) for WMAP compatible models. The $\eta$-parameter is equivalent to the $\eta_{0}$-parameter discussed in Sect. 2.3. The dashed region corresponds to the $2 \sigma \mathrm{CL}$ upper limit from M 31 observations with CELESTE.

support by Antoine Pérez, Jacques Maurand and Stéphane Rivoire at Thémis was invaluable. Last but not least, we appreciatively thank Karsten Jedamzik, Gilbert Moultaka and Pierre Salati for very useful discussions during this work.

\section{References}

Aharonian, F. A., et al. 2003, A\&A, 400, 153

Bennet, C. L. 2003, APJS, 148

Bergström, L. 2000, Rep. Prog. Phys., 63, 793

Bergström, L., Ullio, P., \& Buckley, J. 1998, Astropart. Phys., 9, 137

Bertone, G., Zentner, A. R., \& Silk, J. 2005, Phys. Rev. D, 72, 103517

Blom, J. J., Paglione, T. A. D., Carramiñana, A., et al. 1999, ApJ, 516, 744

Blumenthal, G. R., Faber, S. M., Primack, J. R., \& Rees, M. J. 1984, Nature, 311, 517

Braun, R. 1991, ApJ, 372, 54

Caldwell, R. R., Dave, R., \& Steinhardt, P. J. 1998, Phys. Rev. Lett., 80,1582

Diemand, J., Moore, B., \& Stadel, J. 2005, Nature, 433, 389

Djouadi, A., Kneur, J.-L., \& Moultaka, G. 2002 [arXiv:hep-ph/0211331]

Falvard, A., Giraud, E., Jacholkowska, A., et al. 2004, Astropart. Phys., 20, 467

Fujii, M., \& Ibe, M. 2004, Phys. Rev. D, 69, 035006

Gnedin, O. Y., Kravtsov, A. V., Klypin, A. A., \& Nagai, D. 2004, ApJ, 616,16

Goldberg, H. 1983, Phys. Rev. Lett., 50, 1419

Gondolo, P., \& Silk, J. 1999, Phys. Rev. Lett., 83, 1719

Gondolo, P., Edsjö, J., Ullio, P., et al. 2004, JCAP, 07, 008

Hawkins, E., Maddox, S., Cole, S., et al. 2003, MNRAS, 346, 78

Heck, D., et al. 1998, Report FZKA 6019, http: //www-ik. fzk.de/ corsika/usersguide/corsika_tech.html 
Kleyna, J., Wilkinson, M. I., Evans, N. W., \& Gilmore, G. 2001, ApJ, 563,115

Lee, B. W., \& Weinberg, S. 1977, Phys. Rev. Lett., 39, 165

Loveday, J., et al. 2002, Contemporary Physics, 43, 437

Manseri, H., et al. 2004, International Symposium on High Energy Gamma-Ray Astronomy, Heidelberg. See also her doctoral thesis, École Polytechnique (Palaiseau, March 2004), at http://doc.in2p3.fr/themis/CELESTE/PUB/papers.html

Moore, B., Quinn, T., Governato, F., Stadel, J., \& Lake, G. 1999, MNRAS, 310, 1147

de Naurois, M., Holder, J., Bazer-Bachi, R., et al. 2002, ApJ, 566, 343

Navarro, J. F., Frenk, C. S., \& White, S. D. M. 1996, ApJ, 462, 563

Nilles, H. P. 1984, Phys. Rept., 110, 1

Ostriker, J. P., Peebles, P. J. E., \& Yahil, A. 1974, ApJ, 193, 1

Paré, É., Balauge, B., Bazer-Bachi, R., et al. 2002, NIM A, 490, 71
Piron, F., et al. 2003, 28th ICRC, Tsukuba, vol. 5 (Universal Academy Press), 2607

Salati, P. 2003, Phys. Lett. B, 571, 121

Servant, G., \& Tait, T. M. P. 2003, Nucl. Phys. B, 650, 391

Smith, D., \& Brion, E. 2004, CELESTE collaboration internal note Spergel, D. N., Verde, L., Peiris, H. V., et al. 2003, ApJS, 148, 175S

Stoehr, F., White, S. D. M., Springel, V., et al. 2003, MNRAS, 345, 1313

Tasitsiomi, A., \& Olinto, A. V. 2002, Phys. Rev. D, 66, 083006

de Vaucouleurs, G. 1958, ApJ, 128, 465

Weekes, T. C. 1988, Phys. Rep., 160, 1

Yahiro, M., Mathews, G. J., Ichiki, K., et al. 2002, Phys. Rev. D, 65, 063502

Zwicky, F. 1937, ApJ, 86, 217 